\title{
Topological and electrical control of cardiac differentiation and assembly
}

\author{
Nimalan Thavandiran ${ }^{1,2}$, Sara S Nunes', Yun Xiao ${ }^{1,2}$ and Milica Radisic ${ }^{1,2,3 *}$
}

\begin{abstract}
Tissue engineering has developed many paradigms and techniques on how to best integrate cells and extracellular matrix to create in vitro structures that replicate native tissue. The strategy best suited for building these constructs depends mainly on the target cells, tissues, and organ of interest, and how readily their respective niches can be recapitulated in vitro with available technologies. In this review we examine engineered heart tissue and two techniques that can be used to induce tissue morphogenesis in artificial niches in vitro: engineered surface topology and electrical control of the system. For both the differentiation of stem cells into heart cells and further assembly of these cells into engineered tissues, these two techniques are effective in inducing in vivolike structure and function. Biophysical modulation through the control of topography and manipulation of the electrical microenvironment has been shown to have effects on cell growth and differentiation, expression of mature cardiac-related proteins and genes, cell alignment via cytoskeletal organization, and electrical and contractile properties. Lastly, we discuss the evolution and potential of these techniques, and bridges to regenerative therapies.
\end{abstract}

\section{Introduction}

Heart failure triggered by myocardial infarction is a leading cause of death globally [1]. As the stages of heart disease progress, the likelihood of patient survival decreases; it is therefore critical to intervene with therapeutics as soon as possible in all cases. Currently, heart transplantation is the only known cure for advanced heart failure; however, with the relative deficiency and

\footnotetext{
*Correspondence: m.radisic@utoronto.ca

IInstitute for Biomaterials and Biomedical Engineering, University of Toronto,

Toronto, Ontario, Canada M5S 3G9

Full list of author information is available at the end of the article
}

immediate unavailability of donor hearts [2], this is not a sustainable strategy for the present and future. Regenerative medicine, which integrates the sciences and technologies of stem cells and tissue engineering, has demonstrated promise in alleviating some of these challenges.

Within regenerative medicine, there are many competing therapeutic strategies; however, the common key elements of almost all strategies inevitably focus on replacing or mobilizing the cells in the heart [3]. Although cardiomyocytes (atrial, ventricular, and nodal) are the primary target cell types in the heart, cardiac fibroblasts (structural, and biochemically supportive), and endothelial and smooth muscle cells (which construct vasculature), are also critical for normal heart function. These cells can come from two potential sources, each of which is, in itself, a separate strategy for regeneration of the heart. The first strategy is to stimulate the remaining live cells in the heart, mainly cardiomyocytes and endothelial cells, which have limited proliferative potential, after an episode of myocardial infarction. This is done by the use of small molecules or other exogenous factors delivered systemically or via intra-myocardial injection. The second strategy is to introduce cells into the diseased area of the heart that have been generated ex vivo, either on their own, or with supportive biomaterials and/or supportive factors [4].

Cardiac tissue engineering aims to manipulate the microenvironment cells interact within in order to facilitate cell assembly and build functional tissue with the goal of providing replacements for diseased or damaged native tissues. Additionally, engineered heart tissue may serve as an increasingly accurate in vitro model for studies in normal and diseased heart physiology, as well as drug discovery, validation, and toxicology [5-7]. With the advent of serum-free cardiac differentiation protocols [8-12] comes the ability to generate large quantities of cardiomyocytes derived from human pluripotent stem cell sources for engineered heart tissue. Additionally, cardiomyocyte-specific surface markers have been identified and microfluidic cell separation methods have been advanced that can be used to purify heterogeneous populations [13-15]. 
The adult mammalian heart is composed of a complex and well-integrated mosaic of anatomical modules. The contractile muscle (atria, and ventricles) positioned between the supporting epi- and endocardium, the conduction system (pacemaker nodes, and Purkinje fiber network), and the highly dense vasculature (endothelial and smooth muscle cells) constitute the key elements of the cardiac system, which is the engine for the larger cardiovascular system. During development, complex tissues are formed as pluripotent stem cells differentiate into increasingly specialized cell types. A primary goal of tissue engineering is to recapitulate the conditions occurring during in vivo development in an in vitro setting. To do this effectively, the complete cellular microenvironment (auto-, para-, and juxtracrine signaling, extracellular matrix (ECM) interactions, and electromechanical stimuli) must be quantitatively measured, understood, engineered, and recapitulated experimentally. In the heart, the many cell types form specific integrated structures that contribute to their individual cell and overall organ function. To engineer these cells in the appropriate positions and to temporally give them the correct biochemical, physical, and electrical cues is the overarching goal.

A functional engineered heart tissue requires the following four criteria: 1) aligned syncytium of cardiomyocytes (and stromal cells) with synchronous electromechanical coupling of adequate contractile force; 2) supportive ECM and scaffolding structure to mimic the mechanical and biochemical properties of native tissue; 3) functional microvasculature to provide adequate nutrient and oxygen delivery within a tissue of clinically relevant thickness; and 4) suitable degree of maturation for either successful implantation and host tissue integration or an appropriate in vitro model mimicking adult heart tissue.

Two techniques that have been used to manipulate cells progressing through cardiac differentiation and functional assembly into engineered heart tissue with positive functional effects are 1) control of extracellular surface topology and geometry, and 2) electrical control by stimulation and the use of conductive biomaterials.

\section{The role of extracellular geometry and electrical properties in cells and tissue}

The response of cells to the changes in microenvironmental signals is enabled by biochemical pathways. A change in substrate stiffness, surface topography, tugging force, or the molecular composition of the surrounding ECM is seen by the cell as a biochemical signal via mechanotransduction-mediated ligand receptor interactions. Similarly, a change in electrical charge density on either side of a cell membrane due to external stimulation, or a sudden influx of extracellular ions is also a biochemical signal that the cell can understand. Many studies suggest that these types of signals are just as important as soluble factor-based autocrine and paracrine signaling in influencing cell fate and state [7,16-18].

The Chen and Discher groups have shown the importance of surface topography and substrate stiffness in directing mesenchymal stem cell fate $[19,20]$. The first study, by McBeath and colleagues [20], determined the significance of surface topography by micropatterning cells onto islands of ECM and observing the resulting effects on cell morphology. A connection was then made between cell morphology (round on small micropatterned islands versus spread out and flat on larger islands) and lineage fate. Specifically, spread out and flat cells under cytoskeletal tension were thought to mediate RhoA expression, which if expressed constitutively directed the mesenchymal stem cells into osteoblasts, and if not expressed, as in the non-spread and round cells, directed them into adipocytes [20]. Engler and colleagues [19] studied the effects of substrate stiffness on directing mesenchymal stem cell fate and found that cells cultured on ECM that mimicked native tissue elasticities were directed to that tissue type. For example, mesenchymal stem cells cultured on brain-like ECM differentiated primarily into neurogenic cells, and cells cultured on muscle-like ECM differentiated into myogenic cells.

During heart development, certain key genes have been shown to be critical for normal cell growth and differentiation. One such gene, Wnt11, has been shown to be necessary for patterning an electrical gradient in zebrafish heart [21]. Interestingly, animals with this gene knocked down showed a uniform conduction velocity along the surface of the heart; in normal hearts, however, there were gradual changes in conduction velocity depending on the local area of the propagation. The researchers excluded the possibility for this gradient of electrical coupling due to cellular excitability, connexin localization, tissue geometry and mechanical inputs. Instead, they showed that Wnt11 expression was solely responsible and that it acted via expression of L-type calcium channels, which affected transmembrane calcium ion conductance in the conducting cardiomyocytes [21]. It is important then to note from this study that a linear electrical stimulus and conduction pattern in heart tissue may not be functionally suitable; it is just as important to quantify the spatial distribution and temporal activity of the ion channels that mediate electrical propagation and directly lead to concerted contractile function.

\section{Structuring engineered heart tissue using topographical cues}

It is well known that the architecture of the extracellular environment influences cell behavior at the nano-, 
micro- and macroscale with respect to the expression of cardiac-specific genes and proteins, cytoskeletal structure, morphology, and functionality. The main complexity involved in engineering functional myocardium is related to establishing appropriate structure-function correlation over different scales. Assembly of appropriate structure is required to achieve a desired function, which is characterized by the development of active force (for example, for rat heart, 20 to $50 \mathrm{mN} / \mathrm{mm}^{2}$ ) and impulse propagation (for example, for rat heart, 20 to $25 \mathrm{~cm} / \mathrm{s}$ ) [22], both of which are considered to be two critical functional measurements. At the macroscale, native heart contains elongated myofibers aligned in parallel; the structure enables coordinated contraction of the ventricle and expulsion of blood. At the microscale, adult cardiomyocytes are rod shaped and contain registries of sarcomeres that enable cell contraction in response to electrical signals. At the nanoscale, each sarcomere contains precisely organized sarcomeric proteins (for example, sarcomeric $\alpha$-actin $/ \alpha$-actinin and myosin heavy chain) that enable coordinated contractions of sarcomeres. By simply manipulating the topography of the surface on which cells are adhered to, repeated reports have indicated structural and functional effects pertaining to heart cells.

Kim and colleagues [23] constructed polyethylene glycol hydrogel substratum with anisotropic nanoscale features to mimic the native myocardial ECM. Although the topographic feature sizes in this study (nanoscale) were much smaller than those in previous studies (microscale), the cells still aligned along the direction of the presented topographic cue, showing a nanotopographic cell-substratum interaction for the first time. Distinguished from previous studies on the microscale [24], in which topographical cues were on the order of cell width, enabling the cells to be oriented by confinement, this study showed nanotopographic cell-substratum interaction mimicking nanoscale cell-ECM interaction in vivo, which can also lead to cardiomyocyte orientation. There were no differences in surface treatment amongst the different groups, nor on the grooves versus the ridges of the engineered substratum, and as a result, cells were able to freely spread and adhere over several ridges. Analysis revealed that this alignment was due to the organization of focal adhesion proteins and the cortical cytoskeleton. Interestingly, the dimension of the grooves had an important effect on the cell-substratum interaction: when the grooves were too narrow $(400 \mathrm{~nm}$ in this study), the cell membrane was unable to penetrate deep into the bottom of the grooves; whereas when the grooves were sufficiently wide ( $800 \mathrm{~nm}$ in this study), the cell membrane penetrated deep enough to fill the grooves completely, resulting in a more extensive cell-substratum adhesion. As a result, the cells on $800 \mathrm{~nm}$-wide-patterned substratum experienced stronger contraction-mediated stress, showed an increase in connexin- 43 expression and an increase in conduction velocity of action potentials.

In an early study, Feinberg and colleagues [25] generated two-dimensional muscular thin films by seeding neonatal rat ventricular cardiomyocytes on a polydimethylsiloxane membrane that could be detached from a thermosensitive poly ( $\mathrm{N}$-isopropylacrylamide) substrate. Once detached, the muscular thin film spontaneously adopted a three-dimensional conformation determined by its film properties and the alignment of the cardiomyocytes, including a continuous anisotropic film or an array of discrete muscle fibers [25]. By careful tailoring of the cell alignment pattern, thin-film shape and electrical-stimulation protocol, these cell-covered sheets could be designed to perform tasks such as gripping, pumping, walking and swimming and could generate forces as high as $4 \mathrm{mN}$ per $\mathrm{mm}^{2}$.

High-resolution diffusion tensor magnetic resonance imaging (DTMRI) and microfabrication were combined by Badie and colleagues $[26,27]$ to fabricate cell monolayers that replicate realistic cross-sections of native cardiac tissue. In-plane cardiac fiber directions in native mouse ventricle were first measured by DTMRI and then projected onto two-dimensional pixels to fabricate photomasks. The photomasks were then used to generate polydimethylsiloxane stamps via soft lithography, and to pattern fibronectin on coverslips to guide the local alignment of cultured cardiomyocytes, ultimately yielding a monolayer with replicated cell orientation. This novel method provides an improved platform to study intramural structure-function relationships with one of their recent studies focused on incidence and spatiotemporal characteristics of conduction block [28].

Takahashi and colleagues [29] have built anisotropic cell sheets by patterning hydrophilic (PIPAAm-b-PAcMo) domains onto thermosensitive (PIPAAm) domains in a stripe pattern. During cultivation, normal human dermal fibroblasts were aligned along with the stripe patterns and showed physical and biological properties different to that of isotropic cell sheets: the anisotropic cell sheets showed increased shrinking rates parallel to cell alignment due to the collective orientation of contractile actin fibers. Moreover, the secretion of vascular endothelial growth factor by aligned fibroblasts was increased significantly and the collagen deposited onto fibroblast sheets was anisotropic. This technology together with the cell sheet stacking technique [30] could generate threedimensional complex anisotropic tissue in vitro.

With a well-developed cell entrapment method, Tiburcy and colleagues [31] generated three-dimensional engineered heart tissue (EHT) from neonatal rat cardiomyocytes and observed terminal differentiation and tissuelike cardiomyocyte maturation supported by similar 
morphological and molecular features of EHT- and postnatal heart-derived cardiomyocytes. They also showed that EHT development had similar distinct phases to cardiomyocyte maturation, including 1) a consolidation phase with high levels of apoptosis and ECM degradation, and 2) a maturation phase with myocyte binucleation, rod-shaped cardiomyocyte formation, a shift from fetalskeletal to adult-cardiac actin transcript expression, and ECM build-up.

Engelmayr and colleagues [32] created an accordionlike scaffold using laser boring of a $250 \mu \mathrm{m}$ thick poly(glycerol sebacate) layer. The scaffolds were pretreated with cardiac fibroblasts by rotating culture, followed by seeding of enriched cardiomyocytes under static culture. At the end of cultivation, the authors obtained contractile cardiac grafts with heart cells aligned along the preferred direction and mechanical properties closely resembling those of a native rat right ventricle.

There were interesting findings in a study by Madden and colleagues [33] in which a bimodal scaffold architecture was developed that provided parallel channels and interconnected porous networks at the same time. The parallel channels were designed to develop cardiomyocyte muscle bundles in vitro while the surrounding sphere-templated porous network was intended to improve diffusive mass transfer. The scaffold was first seeded with primary chicken embryonic-derived cardiomyocytes (approximately 20 to 25\% cardiomyocyte purity) by centrifuging cells into the parallel channels. During cultivation, the proliferation of non-myocytes within the porous network and around the scaffold edge decreased the supply of oxygen and nutrients to cardiomyocytes, which principally remained in the channels. Therefore, the viability of cardiomyocytes was limited to within approximately $150 \mu \mathrm{m}$ of the construct surface. However, when the scaffold was seeded with human embryonic stem cell-derived cardiomyocytes (10 to 65\% cardiomyocytes), non-myocytes declined over a 5-day cultivation period, resulting in predominantly cardiomyocytes (approximately 95\% $\beta$-myosin heavy chainpositive) in the cell population and porous channel walls free of cells. Because of the improved mass transfer, the cell survival was increased up to $300 \mu \mathrm{m}$ into the scaffold. The mechanism responsible for the decrease in the nonmyocyte fraction within this scaffold is not entirely clear; however, it is likely related to the unique three-dimensional structure.

Understanding the mechanisms associated with topology-based signaling in two dimensions will certainly have implications in three-dimensional tissue engineering. Currently, however, there is a lack of established technologies that will permit three-dimensional topological patterning inside three-dimensional matrices such as hydrogels. It is clear that cells are affected by topology, but to preserve distinct topologies in engineered threedimensional substrates containing embedded cells that remain viable requires sophisticated technologies such as three-dimensional printing capabilities, and hydrogel post-polymerization techniques, both of which need to occur at high resolution in the nanometre range. Therefore, current two-dimensional studies help determine favorable geometries of topology that may transfer well into three-dimensional systems once appropriate technologies are developed. Additionally, these studies can provide great bases for computational models that can be designed to simulate three-dimensional tissue topographies.

\section{Electrical control of engineered heart tissue}

During embryo development, cells are exposed not only to gradients of soluble factors but also to endogenous electrical fields that may determine the emergence of spatial patterns and aid in tissue morphogenesis [34]. Exogenously applied electrical stimulation has been shown to also influence cell behavior [35]. In the cardiac development context, electrical field stimulation has been shown to affect the differentiation of mouse embryonic stem cells in vitro [36]. In the study by Sauer and colleagues [36], a single direct current field pulse was applied to 4-day-old embryoid bodies and the authors found significant effects of pulses applied for 90 seconds on cardiomyocyte differentiation with field strengths of 250 and $500 \mathrm{~V} / \mathrm{m}$. This electrical stimulation protocol increased both the number of differentiating beating embryoid body foci as well as the size of the beating foci. A comparable increase in the number of beating embryoid bodies was achieved by incubation with $\mathrm{H}_{2} \mathrm{O}_{2}$, indicating that the electrical field effect was transduced via the intracellular generation of reactive oxygen species. The radical scavengers dehydroascorbate and pyrrolidinedithiocarbamate, and the NF-kB antagonist N-tosyl-Lphenylalanine chloromethyl ketone inhibited cardiac differentiation, suggesting that reactive oxygen species and NF-kB may play a role in early cardiac development. Electrical stimulation has also been shown to play a role in cardiac differentiation of human embryonic stem cells [37], through mechanisms associated with the intracellular generation of reactive oxygen species. In the cardiac tissue engineering context, electrical field stimulation has been used to improve tissue properties [38-41]. After 24 hours of regular electrical stimulation of adult ventricular myocytes in culture, cells displayed higher caffeine-induced $\mathrm{Ca}^{2+}$ transients than non-stimulated controls [40]. Field stimulation also enhanced the mechanical properties of myocytes when compared to quiescent myocytes, suggesting that regular electrical stimulation is important when studying the function of adult ventricular myocytes in culture. 
Radisic and colleagues [41] have shown that the application of electrical stimulation during construct cultivation markedly enhanced the contractile behavior of rat neonatal cardiomyocytes cultured on scaffolds. There was also a decrease in the excitation threshold and an increase in maximum capture rate both with time and with electrical stimulation. Analysis of cardiomyocyte ultrastructure revealed that myofibrils aligned in the direction of electrical field lines [41] and promoted a remarkable level of ultrastructural organization in threedimensional tissues. Importantly, it was shown that if applied early after seeding (day 1), electrical stimulation inhibited the accumulation of cardiac proteins and yielded poor contractile behavior. If applied late (day 5), electrical stimulation was less effective because of the reduced amounts of connexin- 43 and contractile proteins available in the cells [41], suggesting that there is a window where electrical stimulation can yield more favorable results.

The effects of monophasic or biphasic electrical field stimulation on the structure and function of engineered cardiac organoids was also studied and shown to yield different results [38]. Field stimulation using symmetric biphasic square pulses was an improved stimulation protocol compared to no stimulation and stimulation using monophasic square pulses of identical total amplitude and duration. This was demonstrated by the highest success rate for synchronous contractions, lower excitation threshold, higher density, and higher expression of connexin-43 in the biphasic group compared to the monophasic group. Biphasic field stimulation was also effective at improving electrical excitability of multicellular type cardiac organoids where fibroblasts and/or endothelial cells were also added [38].

Electrical stimulation can also be combined with bioreactor perfusion to generate thick, functional cardiac patches [42]. Bioreactor cultivation for 4 days under perfusion with continuous electrical stimulation promoted elongation and striation of rat neonatal cardiomyocytes and increased expression of connexin-43 [42]. This illustrates the effectiveness of electrical field stimulation even in a rather complex cultivation system such as a perfusion bioreactor. Electrical stimulation has also been shown to significantly increase the average conduction velocity of neonatal rat cardiomyocyte constructs [43], which correlated with the improved contractile behavior of tissue constructs. Electrical stimulation during culture significantly improved amplitude of contractions, tissue morphology, and connexin- 43 expression compared to the non-simulated controls [43].

Taken together, these reports demonstrate the benefits of electrical stimulation to cardiac tissue engineering in animal models. To date, however, there are no reports in the literature of the effects of electrical field stimulation in human cardiac tissue engineering.

\section{Interactive effects of topographical and electrical cues}

A small number of studies have focused on evaluating the interactive effects of topography and electrical field stimulation. When both cues are simultaneously applied, an interesting study is to determine which of the two will preferentially guide the cell orientation and elongation response as well as determine the cell phenotype. In a related study, interactive effects were investigated using pulsatile electrical field stimulation and substrates with approximately $700 \mathrm{~nm}$ deep 'V'-shaped abrasions [44]. Although both fibroblasts and cardiomyocytes elongated and aligned on non-abraded surfaces by application of electrical field stimulation, topographical cues were a significantly stronger determinant of cardiomyocyte orientation than the electrical field stimulation. The orientation and elongation response of cardiomyocytes was completely abolished by inhibition of actin polymerization (cytochalasin D) and only partially by inhibition of the phosphatidyl-inositol 3 kinase (PI3K) pathway (LY294002).

In a subsequent set of related studies, precise topographical cues were engineered by hot embossing tissue culture polystyrene with defined microgrooves and microridges [45]. The electrical stimulation electrodes were deposited on the chip edges such that the grooves were oriented either parallel or perpendicular to the field lines. Substrates consisted of $0.5 \mu \mathrm{m}$-wide grooves and $0.5 \mu \mathrm{m}$-wide ridges $(1 \mu \mathrm{m}$ period) or $3 \mu \mathrm{m}$-wide grooves and $1 \mu \mathrm{m}$-wide ridges $(4 \mu \mathrm{m}$ period); in all cases the grooves were $400 \mathrm{~nm}$ deep and the smooth substrates were used as controls. Neonatal rat cardiomyocytes elongated and aligned along the microgrooves forming a welldeveloped contractile apparatus, staining positively for sarcomeric $\alpha$-actinin, with a more pronounced effect on substrates with $1 \mu \mathrm{m}$ compared to $4 \mu \mathrm{m}$ periodicity. Importantly, simultaneous application of biphasic electrical pulses and topographical cues resulted in gap junctions confined to the cell-cell end junctions rather than the punctate distribution found in neonatal cells. Electrical field stimulation further enhanced cardiomyocyte elongation when microgrooves were oriented parallel to the electric field lines.

By incorporating gold nanowires within alginate scaffolds, Dvir and colleagues [46] were able to increase the conductivity of this biomaterial and improve electrical communication between adjacent cardiac cells. Tissues grown on these composite matrices were thicker and better aligned than those grown on pristine alginate. In addition, higher levels of the proteins involved in muscle contraction and electrical coupling were detected in the composite matrices. When subjected to electrical stimulation, the cells in these tissues contracted synchronously. 
Tandon and colleagues described a novel surfacepatterned microbioreactor array, where an excimer laserbased method was used to generate a micropatterned indium tin oxide substrate with an interdigitated array of electrodes designed for electrical stimulation of cultured cells. The excimer laser-based method enables direct patterning of the indium tin oxide in a single step, and without the use of harsh chemicals or a customized photomask. This allowed for the generation of a patternable and optical imaging-compatible substrate for longterm, microscale cell culture with electrical stimulation [47]. The system has been used to culture primary cardiomyocytes and human adipose-derived stem cells. Over 6 days of culture with electrical stimulation $(2 \mathrm{~ms}$ duration, $1 \mathrm{~Hz}, 180 \mu \mathrm{m}$ wide electrodes with $200 \mu \mathrm{m}$ spacing), both cell types exhibited enhanced proliferation, elongation and alignment, and adipose-derived stem cells exhibited higher numbers of connexin-43-composed gap junctions.

\section{Perspectives}

It is clear that much work and development is required to advance the field of stem cell and cardiac tissue engineering to the point of significant clinical impact. The emerging technologies within the fields of biology, material science, micro- and nano-fabrication, and computational modeling are all progressing at a rapid pace. The challenge, however, is choosing the correct combination of technologies married with suitable biology to create human tissue replacements and in vivolike in vitro models that are functional.

In the context of microenvironmental control in the heart, it is necessary to mention the importance of the dynamic contractile forces that are present. The ECM plays a critical role in the heart cell niche during development, homeostasis, disease, and repair. One primary mode in which the ECM communicates with heart cells is through mechanotransductive cues. Aside from static biomechanical cues (facilitated by cell integrins and focal adhesions) dynamic cues that provide stretching forces to cells through the ECM have been shown to be important in heart development and maturation. The Eschenhagen and Zimmerman groups have investigated and reported on the role and beneficial effects of mechanical stimulation in cardiac cells $[31,48-50]$. External mechanical stimulation aims to recapitulate the electromechanical forces observed regularly in the contracting native heart. Much like electrical stimulation, mechanical stimulation directs the elongation and orientation of cardiomyocytes, in addition to improving force of contraction and stage of maturation. Electrical stimulation may, however, be a more physiological (albeit indirect) method of inducing mechanical stimulation (compared to stretching) as this occurs in vivo via excitation-contraction coupling.
Two methods that hold promise in generating mature engineered heart tissue are 1) the control of geometrical cues and 2) the manipulation of electrical properties within the cellular microenvironment. Figure 1 summarizes the main concepts discussed and how they link to downstream effects leading eventually to changes in function. Future development will likely bring interesting advances and marriages of the mentioned concepts; in fact, there is evidence for some aspects of this research ongoing currently.

Computational modeling is often underutilized in tissue engineering. Recent advances in the sophistication and complexity of theoretical mechanotransduction models, in addition to empirical techniques with which to validate models, have made these approaches a rich source of insight and predictability (reviewed in [51]). The end function of heart muscle is to contract at a force and rate appropriate for blood circulation. The contractility of cardiomyocytes has been modeled by numerous groups. In a recent study, Shim and colleagues [52] developed a model system that can detect the force of contraction exerted by a monolayer. Cardiomyocytes were seeded onto a thin film that curled in response to the force of contraction of adhered cardiomyocytes. The magnitude of exerted force was calculated by the degree of curvature of the thin film. In order to determine optimized designs for their model, they developed a finite element-based three-dimensional phenomenological constitutive model, which accounted for both the passive deformation, including pre-stretch, and the active behavior of the cardiomyocytes.

One notion that may prove useful in screening studies is a surrogate system for EHT that has the capability not only to provide the correct control cues for heart development and maturation, but also to simultaneously sense tissue function. This is currently a key obstacle for model system development, especially for a system that attempts to integrate a tissue mimetic (as opposed to two-dimensional monolayer culture) in a high-content and high-throughput manner. A few groups have utilized polymer-based cantilever systems to culture miniature tissues that simultaneously restrain the remodeling of tissue and report forces exerted [18,49,50,53]. It would be interesting to integrate electrical control with these types of systems to both stimulate and record electrical activity while maintaining appropriate force dynamics. A system like this would constitute a complete model whereby form and function of engineered heart tissue could be controlled and sensed concurrently.

In vivo, cells are able to communicate and self-assemble without much difficulty. Self-assembly in vitro has always been a desirable option for tissue engineers, although it has proven difficult to recapitulate key signals present in vivo that influence cells to build appropriate structure 


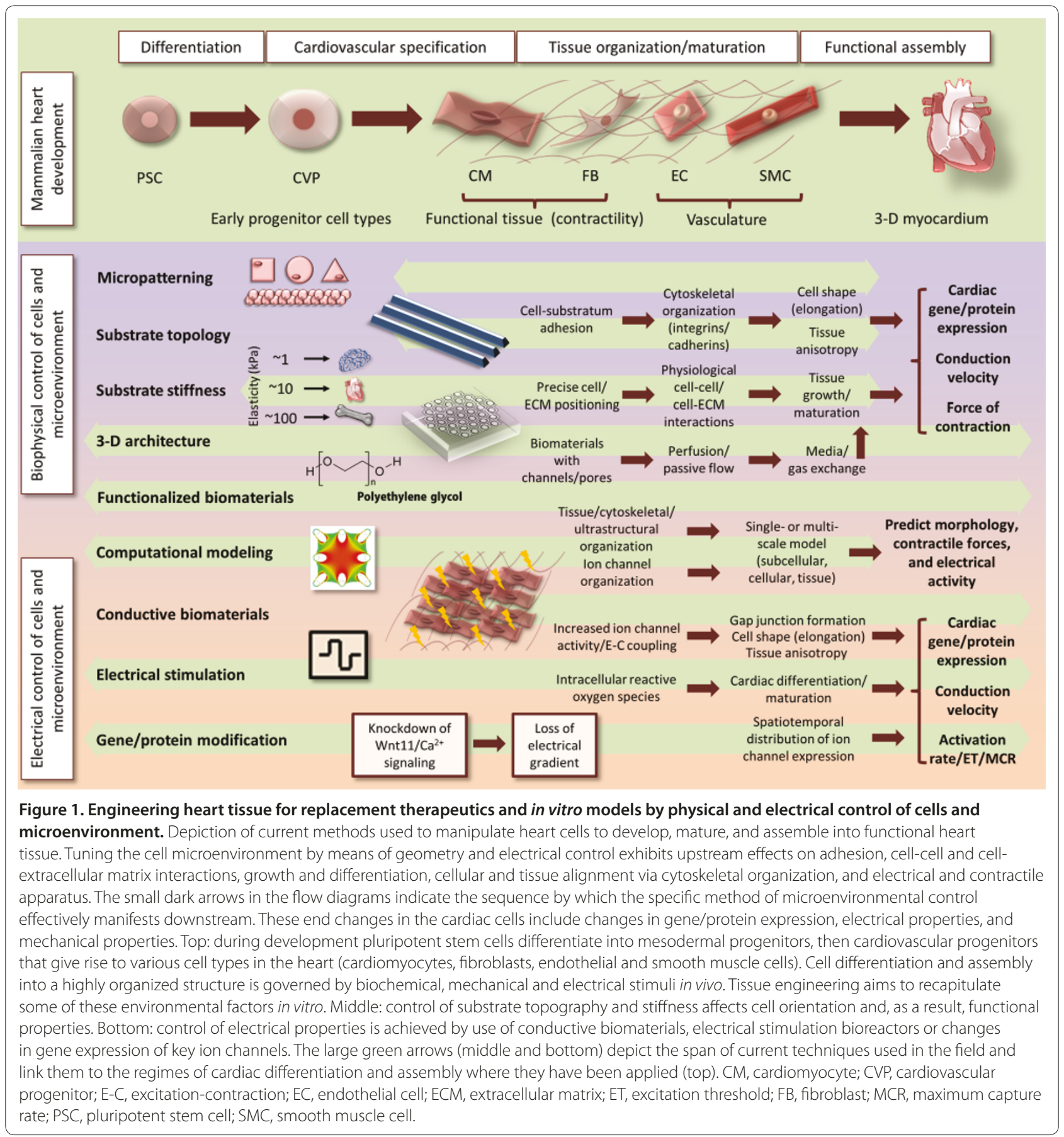

and associated function. Recapitulation of tissue morphogenesis by inducing self-organization in vitro has so far been demonstrated in many organ subunits, including the eye [54], liver [55], intestine [56], and brain [57], although not yet in the heart. This is a highly promising method of inducing tissue morphogenesis in parallel with directed cardiac differentiation, and may be supplemented with biophysical and electrical control of the microenvironment. The next generation of engineered heart tissue should take further advantage of the intrinsic self-assembly and self-organization capabilities of cells with the aid of external electrical and mechanical cues to facilitate functional tissue construction. This bottom up approach to tissue engineering may prove efficient, provided the microenvironment can be accurately recapitulated. 


\section{Conclusion}

When guiding the differentiation of human pluripotent stem cells into heart cells, recapitulating key factors found in the native environment of the cardiac niche is critical. In addition to biochemical factors, it is necessary to integrate appropriate topology and electrical control of the system to enable the assembly of functional cardiac tissue. Engineered human heart tissue that has the capability to mimic the mature molecular signature and physiology of adult heart tissue will prove to be critical in drug testing applications, studies in cardiac pathophysiology, and development of new cell replacement therapies.

This article is part of a thematic series on Physical influences on stem cells edited by Gordana Vunjak-Novakovic. Other articles in the series can be found online at http://stemcellres.com/series/physical

\section{Abbreviations}

DTMRI, diffusion tensor magnetic resonance imaging; ECM, extracellular matrix; EHT, engineered heart tissue; $\mathrm{NF}$, nuclear factor.

\section{Acknowledgements}

The authors would like to acknowledge the funding sources: NSERC Discovery Grant (RGPIN 326982-10), NSERC Strategic Grant (STPGP 381002-09), NSERCCIHR Collaborative Health Research Grant (CHRPJ 385981-10), HSFO GrantIn-Aid (T6946) and Discovery Accelerator Supplement (RGPAS 396125-10). Milica Radisic is the Canada Research Chair in Functional Cardiovascular Tissue Engineering. Nimalan Thavandiran receives funding from a HSFC/CIHR Doctoral Research Award and from a NSERC Microfluidic Applications and Training in Cardiovascular Health (MATCH) Scholarship.

\section{Competing interests}

The authors declare that they have no competing financial interests.

\section{Author details}

${ }^{1}$ Institute for Biomaterials and Biomedical Engineering, University of Toronto, Toronto, Ontario, Canada M5S 3G9. ${ }^{2}$ Department of Chemical Engineering and Applied Chemistry, University of Toronto, Toronto, Ontario, Canada M5S 3 G9. ${ }^{3}$ Heart and Stroke/Richard Lewar Centre of Excellence, University of Toronto, Toronto, Ontario, Canada M5S 3G9.

Published: 14 February 2013

\section{References}

1. Bui A, Horwich T, Fonarow G: Epidemiology and risk profile of heart failure. Nat Rev Cardiol 2011, 8:30-41.

2. Stehlik J, Edwards L, Kucheryavaya A, Benden C, Christie J, Dobbels F, Kirk R, Rahmel A, Hertz M: The Registry of the International Society for Heart and Lung Transplantation: Twenty-eighth Adult Heart Transplant Report--2011.J Heart Lung Transplant 2011, 30:1078-1094.

3. Vunjak-Novakovic G, Tandon N, Godier A, Maidhof R, Marsano A, Martens T, Radisic M: Challenges in cardiac tissue engineering. Tissue Eng Part B Rev 2010, 16:169-187.

4. Ptaszek L, Mansour M, Ruskin J, Chien K: Towards regenerative therapy for cardiac disease. Lancet 2012, 379:933-942.

5. Dengler J, Song H, Thavandiran N, Massé S, Wood G, Nanthakumar K, Zandstra P, Radisic M: Engineered heart tissue enables study of residual undifferentiated embryonic stem cell activity in a cardiac environment. Biotechnol Bioeng 2011, 108:704-719.

6. Song H, Yoon C, Kattman S, Dengler J, Massé S, Thavaratnam T, Gewarges M, Nanthakumar K, Rubart M, Keller G, Radisic M, Zandstra P: Interrogating functional integration between injected pluripotent stem cell-derived cells and surrogate cardiac tissue. Proc Natl Acad Sci U S A 2010, 107:3329-3334

7. Song H, Zandstra P, Radisic M: Engineered heart tissue model of diabetic myocardium. Tissue Eng Part A 2011, 17:1869-1878.
8. Kattman S, Witty A, Gagliardi M, Dubois N, Niapour M, Hotta A, Ellis J, Keller G Stage-specific optimization of activin/nodal and BMP signaling promotes cardiac differentiation of mouse and human pluripotent stem cell lines. Cell Stem Cell 2011, 8:228-240.

9. Bauwens C, Song H, Thavandiran N, Ungrin M, Massé S, Nanthakumar K, Seguin C, Zandstra P: Geometric control of cardiomyogenic induction in human pluripotent stem cells. Tissue Eng Part A 2011, 17:1901-1909.

10. Murry C, Keller G: Differentiation of embryonic stem cells to clinically relevant populations: lessons from embryonic development. Cell 2008, 132:661-680.

11. Lian X, Hsiao C, Wilson G, Zhu K, Hazeltine L, Azarin S, Raval K, Zhang J, Kamp T, Palecek S: Robust cardiomyocyte differentiation from human pluripotent stem cells via temporal modulation of canonical Wnt signaling. Proc Natl Acad SciU S A 2012, 109:57.

12. Xu C, Police S, Hassanipour M, Li Y, Chen Y, Priest C, O'Sullivan C, Laflamme M, Zhu W-Z, Van Biber B, Hegerova L, Yang J, Delavan-Boorsma K, Davies A, Lebkowski J, Gold J: Efficient generation and cryopreservation of cardiomyocytes derived from human embryonic stem cells. Regen Med 2011, 6:53-66.

13. Dubois N, Craft A, Sharma P, Elliott D, Stanley E, Elefanty A, Gramolini A, Keller $\mathrm{G}: \mathrm{SIRPA}$ is a specific cell-surface marker for isolating cardiomyocytes derived from human pluripotent stem cells. Nat Biotechnol 2011, 29:1011-1018.

14. Uosaki H, Fukushima H, Takeuchi A, Matsuoka S, Nakatsuji N, Yamanaka S, Yamashita J: Efficient and scalable purification of cardiomyocytes from human embryonic and induced pluripotent stem cells by VCAM1 surface expression. PloS one 2011, 6:e23657.

15. Zhang B, Green JV, Murthy SK, Radisic M: Label-free enrichment of functional cardiomyocytes using microfluidic deterministic lateral flow displacement. PLoS One 2012, 7:e37619.

16. Chiu L, Janic K, Radisic M: Engineering of oriented myocardium on threedimensional micropatterned collagen-chitosan hydrogel. Int J ArtifOrgans 2012, 35:237-250.

17. Bhana B, lyer R, Chen W, Zhao R, Sider K, Likhitpanichkul M, Simmons C, Radisic M: Influence of substrate stiffness on the phenotype of heart cells. Biotechnol Bioeng 2010, 105:1148-1160.

18. Boudou T, Legant W, Mu A, Borochin M, Thavandiran N, Radisic M, Zandstra P Epstein J, Margulies K, Chen C: A microfabricated platform to measure and manipulate the mechanics of engineered cardiac microtissues. Tissue Eng Part A 2012, 18:910-919.

19. Engler A, Sen S, Sweeney H, Discher D: Matrix elasticity directs stem cell lineage specification. Cell 2006, 126:677-689.

20. McBeath R, Pirone D, Nelson C, Bhadriraju K, Chen C: Cell shape, cytoskeletal tension, and RhoA regulate stem cell lineage commitment. Dev Cell 2004, 6:483-495

21. Panáková $D$, Werdich $A$, Macrae $C$ : Wnt11 patterns a myocardial electrical gradient through regulation of the L-type $\mathrm{Ca}(2+)$ channel. Nature 2010, 466:874-878.

22. Zimmermann WH, Didie M, Doker S, Melnychenko I, Naito H, Rogge C Tiburcy M, Eschenhagen T: Heart muscle engineering: An update on cardiac muscle replacement therapy. Cardiovasc Res 2006, 71:419-429.

23. Kim D-H, Lipke E, Kim P, Cheong R, Thompson S, Delannoy M, Suh K-Y, Tung L, Levchenko A: Nanoscale cues regulate the structure and function of macroscopic cardiac tissue constructs. Proc Natl Acad Sci U S A 2010, 107:565-570.

24. Bursac N, Parker K, Iravanian S, Tung L: Cardiomyocyte cultures with controlled macroscopic anisotropy: a model for functional electrophysiological studies of cardiac muscle. Circ Res 2002, 91:54.

25. Feinberg AW, Feigel A, Shevkoplyas SS, Sheehy S, Whitesides GM, Parker KK: Muscular thin films for building actuators and powering devices. Science 2007, 317:1366-1370.

26. Badie N, Bursac N: Novel micropatterned cardiac cell cultures with realistic ventricular microstructure. Biophys J 2009, 96:3873-3885.

27. Badie N, Satterwhite L, Bursac N: A method to replicate the microstructure of heart tissue in vitro using DTMRI-based cell micropatterning. Ann Biomed Eng 2009, 37:2510-2521.

28. Badie N, Scull J, Klinger R, Krol A, Bursac N: Conduction block in micropatterned cardiomyocyte cultures replicating the structure of ventricular cross-sections. Cardiovasc Res 2012, 93:263-271.

29. Takahashi H, Nakayama M, Shimizu T, Yamato M, Okano T: Anisotropic cell sheets for constructing three-dimensional tissue with well-organized cell 
orientation. Biomaterials 2011, 32:8830-8838

30. Sasagawa T, Shimizu T, Sekiya S, Haraguchi Y, Yamato M, Sawa Y, Okano T: Design of prevascularized three-dimensional cell-dense tissues using a cell sheet stacking manipulation technology. Biomaterials 2010 31:1646-1654.

31. Tiburcy M, Didié M, Boy O, Christalla P, Döker S, Naito H, Karikkineth B, El-Armouche A, Grimm M, Nose M, Eschenhagen T, Zieseniss A, Katschinksi D, Hamdani N, Linke W, Yin X, Mayr M, Zimmermann W-H: Terminal differentiation, advanced organotypic maturation, and modeling of hypertrophic growth in engineered heart tissue. Circ Res 2011, 109:1105-1114.

32. Engelmayr $\mathrm{G}$, Cheng M, Bettinger C, Borenstein J, Langer R, Freed L: Accordion-like honeycombs for tissue engineering of cardiac anisotropy. Nat Materials 2008, 7:1003-1010

33. Madden L, Mortisen D, Sussman E, Dupras S, Fugate J, Cuy J, Hauch K, Laflamme M, Murry C, Ratner B: Proangiogenic scaffolds as functional templates for cardiac tissue engineering. Proc Natl Acad Sci U S A 2010 107:15211-15216.

34. Nuccitelli R: Endogenous ionic currents and DC electric fields in multicellular animal tissues. Bioelectromagnetics 1992, Suppl 1:147-157.

35. Zhao M, Forrester J, McCaig C: A small, physiological electric field orients cell division. Proc Natl Acad Sci U S A 1999, 96:4942-4946.

36. Sauer H, Rahimi G, Hescheler J, Wartenberg M: Effects of electrical fields on cardiomyocyte differentiation of embryonic stem cells. J Cell Biochem 1999, 75:710-723.

37. Serena E, Figallo E, Tandon N, Cannizzaro C, Gerecht S, Elvassore N, VunjakNovakovic G: Electrical stimulation of human embryonic stem cells: cardiac differentiation and the generation of reactive oxygen species. Exp Cell Res 2009, 315:3611-3619.

38. Chiu L, lyer R, King J-P, Radisic M: Biphasic electrical field stimulation aids in tissue engineering of multicell-type cardiac organoids. Tissue Eng Part A 2011, 17:1465-1477

39. Nunes S, Song H, Chiang C, Radisic M: Stem cell-based cardiac tissue engineering. J Cardiovasc Trans/ Res 2011, 4:592-602.

40. Holt E, Lunde P, Sejersted O, Christensen G: Electrical stimulation of adult rat cardiomyocytes in culture improves contractile properties and is associated with altered calcium handling. Basic Res Cardio/ 1997, 92:289-298

41. Radisic M, Park H, Shing H, Consi T, Schoen F, Langer R, Freed L, VunjakNovakovic G: Functional assembly of engineered myocardium by electrical stimulation of cardiac myocytes cultured on scaffolds. Proc Natl Acad Sci USA 2004, 101:18129-18134

42. Barash Y, Dvir T, Tandeitnik P, Ruvinov E, Guterman H, Cohen S: Electric field stimulation integrated into perfusion bioreactor for cardiac tissue engineering. Tissue Eng Part C Methods 2010, 16:1417-1426.

43. Radisic M, Fast V, Sharifov O, Iyer R, Park H, Vunjak-Novakovic G: Optical mapping of impulse propagation in engineered cardiac tissue. Tissue Eng Part A 2009, 15:851-860.

44. Au HT, Cheng I, Chowdhury MF, Radisic M: Interactive effects of surface topography and pulsatile electrical field stimulation on orientation and elongation of fibroblasts and cardiomyocytes. Biomaterials 2007, 28:4277-4293

45. Au P, Tam J, Fukumura D, Jain RK: Bone marrow-derived mesenchymal stem cells facilitate engineering of long-lasting functional vasculature. Blood 2008, 111:4551-4558.

46. Dvir T, Timko B, Brigham M, Naik S, Karajanagi S, Levy O, Jin H, Parker K, Langer R, Kohane D: Nanowired three-dimensional cardiac patches. Nat Nanotechnol 2011, 6:720-725.

47. Tandon N, Marsano A, Maidhof R, Numata K, Montouri-Sorrentino C, Cannizzaro C, Voldman J, Vunjak-Novakovic G: Surface-patterned electrode bioreactor for electrical stimulation. Lab Chip 2010, 10:692-700.

48. Schaaf S, Shibamiya A, Mewe M, Eder A, Stöhr A, Hirt M, Rau T, Zimmermann W-H, Conradi L, Eschenhagen T, Hansen A: Human engineered heart tissue as a versatile tool in basic research and preclinical toxicology. PloS one 2011, 6:e26397.

49. Hansen A, Eder A, Bönstrup M, Flato M, Mewe M, Schaaf S, Aksehirlioglu B, Schwoerer A, Schwörer A, Uebeler J, Eschenhagen T: Development of a drug screening platform based on engineered heart tissue. Circ Res 2010, 107:35-44.

50. Eschenhagen T, Eder A, Vollert I, Hansen A: Physiological aspects of cardiac tissue engineering. Am J Physiol Heart Circ Physiol 2012, 303:43.

51. Sheehy S, Grosberg A, Parker K: The contribution of cellular mechanotransduction to cardiomyocyte form and function. Biomech Model Mechanobiol 2012, 11:1227-1239.

52. Shim J, Grosberg A, Nawroth J, Parker K, Bertoldi K: Modeling of cardiac muscle thin films: pre-stretch, passive and active behavior. J Biomech 2012, 45:832-841.

53. Vandenburgh H, Shansky J, Benesch-Lee F, Barbata V, Reid J, Thorrez L, Valentini R, Crawford G: Drug-screening platform based on the contractility of tissue-engineered muscle. Muscle Nerve 2008, 37:438-447.

54. Nakano T, Ando S, Takata N, Kawada M, Muguruma K, Sekiguchi K, Saito K, Yonemura S, Eiraku M, Sasai Y: Self-formation of optic cups and storable stratified neural retina from human ESCs. Cell Stem Cell 2012, 10:771-785.

55. Takebe T, Sekine K, Suzuki Y, Enomura M, Tanaka S, Ueno Y, Zheng YW, Taniguchi H: Self-organization of human hepatic organoid by recapitulating organogenesis in vitro. Transplant Proc 2012, 44:1018-1020

56. Spence J, Mayhew C, Rankin S, Kuhar M, Vallance J, Tolle K, Hoskins E, Kalinichenko V, Wells S, Zorn A, Shroyer N, Wells J: Directed differentiation of human pluripotent stem cells into intestinal tissue in vitro. Nature 2011, 470:105-109.

57. Suga H, Kadoshima T, Minaguchi M, Ohgushi M, Soen M, Nakano T, Takata N, Wataya T, Muguruma K, Miyoshi H, Yonemura S, Oiso Y, Sasai Y: Self-formation of functional adenohypophysis in three-dimensional culture. Nature 2011, 480:57-62

\section{doi:10.1186/scrt162}

Cite this article as: Thavandiran N, et al:: Topological and electrical control of cardiac differentiation and assembly. Stem Cell Research \& Therapy 2013, 4:14. 